\title{
Surgical Treatment for Mediastinal Lymph Node Carcinoma of Unknown Primary
}

\author{
Masao Saito*, Tatsuo Nakagawa, Naohisa Chiba, Yasuto Sakaguchi, Shinya Ishikawa \\ Department of Thoracic Surgery, Tenri Hospital, Nara, Japan \\ Email: "msaito@tenriyorozu.jp
}

Received 6 October 2014; revised 6 November 2014; accepted 28 November 2014

Copyright (C) 2014 by authors and Scientific Research Publishing Inc.

This work is licensed under the Creative Commons Attribution International License (CC BY). http://creativecommons.org/licenses/by/4.0/

(c) (i) Open Access

\begin{abstract}
Carcinoma of unknown primary (CUP) is occasionally encountered in clinical oncology. Wide variation exists in CUP. We herein report a rare case of CUP of a mediastinal lymph node. A 61-year-old man with dermatomyositis was referred to our hospital for treatment of mediastinal adenopathy and gastric cancer. Biopsy of both lesions showed that they were histologically different from each other. Mediastinal lymphadenectomy and total gastrectomy were performed for a definitive diagnosis and radical cure. Pathological examination revealed two distinctly different disease processes. The patient underwent postoperative chemotherapy for residual gastric cancer. Twenty months postoperatively, he is alive with cancer. Although CUP usually has a poor prognosis, surgical treatment of metastatic mediastinal lymph node CUP is a feasible therapeutic option.
\end{abstract}

\section{Keywords}

Carcinoma of Unknown Primary (CUP), Mediastinal Lymph Node Carcinoma, Surgical Treatment

\section{Introduction}

Carcinoma of unknown primary (CUP) is defined as biopsy-proven metastasis from a malignancy in the absence of an identifiable primary site after a thorough medical evaluation. CUP constitutes as many as 3\% to 5\% of all cancer diagnoses [1]. CUP represents a heterogeneous group of cancer, and affected patients are divided into subsets of those with a favorable prognosis (20\%) and those with an unfavorable prognosis (80\%) [1].

Metastatic mediastinal lymph node CUP is a rare subset of CUP. It is not included in the favorable subset, but its prognosis is better than that in the unfavorable subset [2]. The optimal treatment strategy of this CUP subset has not yet been confirmed.

\footnotetext{
${ }^{*}$ Corresponding author.
}

How to cite this paper: Saito, M., Nakagawa, T., Chiba, N., Sakaguchi, Y. and Ishikawa, S. (2014) Surgical Treatment for Mediastinal Lymph Node Carcinoma of Unknown Primary. Open Journal of Thoracic Surgery, 4, 90-93. 


\section{Case Report}

A 61-year-old man with dermatomyositis was referred to our hospital because of mediastinal lymph node adenopathy that had been detected by computed tomography (CT). The CT scan revealed a round, 3-cm-diameter mass in the upper mediastinum (Figure 1). Whole-body positron emission tomography/CT revealed abnormal accumulation of substrate within the mediastinal tumor and greater curvature of the stomach (Figure 2).

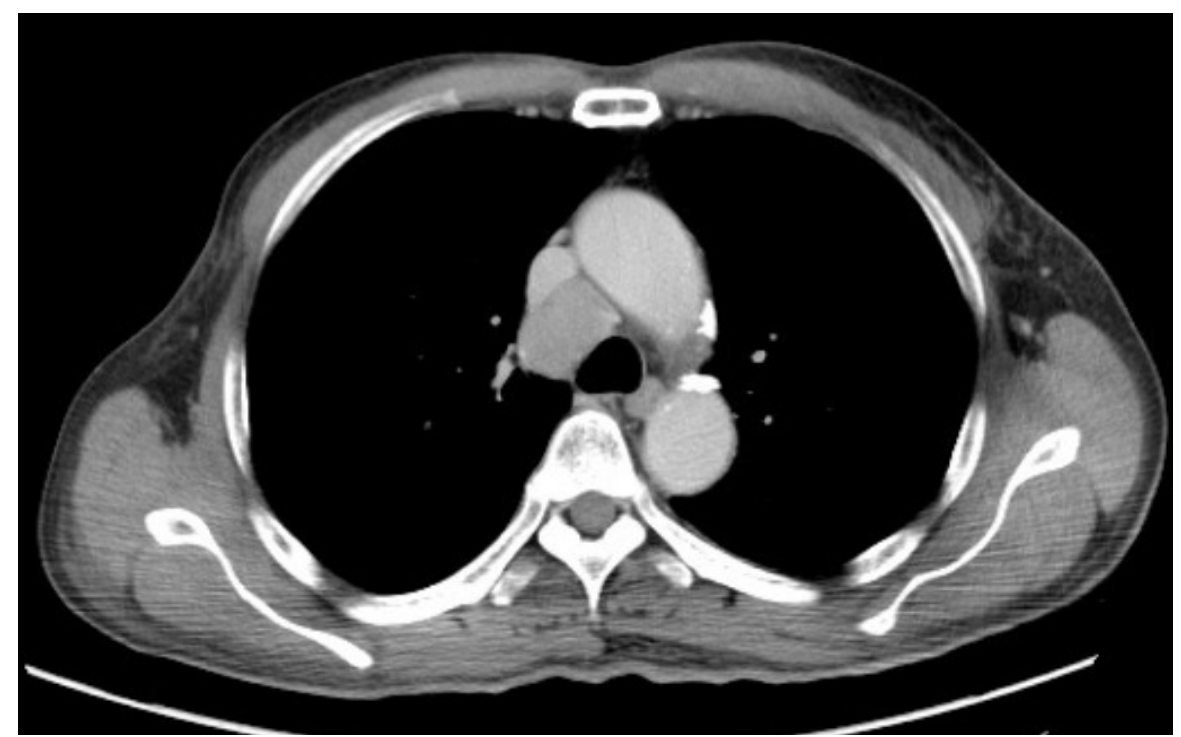

Figure 1. Computed tomography scan showing mediastinal lymph node adenopathy.
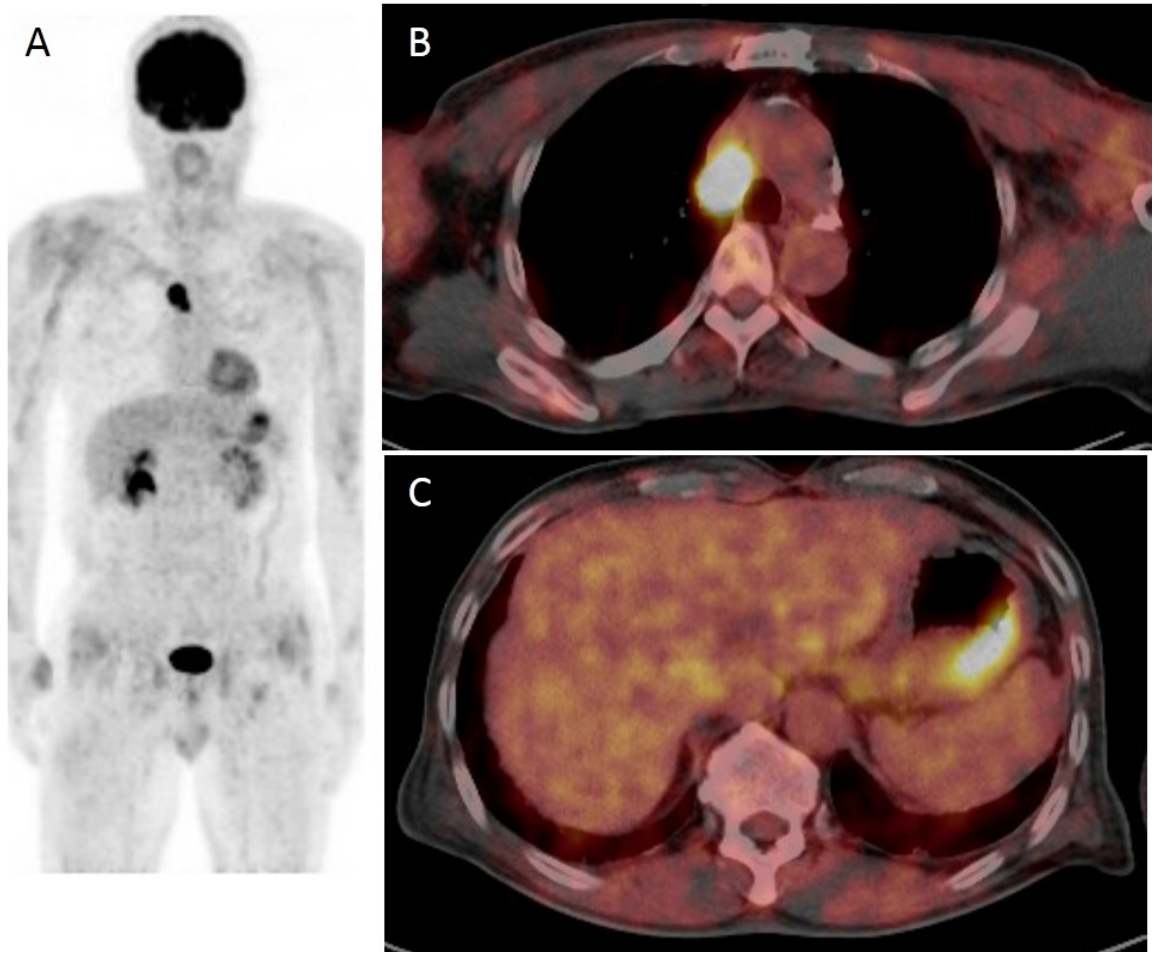

Figure 2. (A) Whole-body positron emission tomography/computed tomography revealed abnormal accumulation of substrate in the mediastinal tumor and greater curvature of the stomach; (B) The maximum standard uptake value in the mediastinal tumor was 12.0; (C) The maximum standard uptake value in the greater curvature of stomach was 7.6. 
The patient underwent upper gastrointestinal endoscopy and biopsy. The pathological findings of the stomach specimen were consistent with adenocarcinoma. He also underwent a transbronchial biopsy of the mediastinal tumor, and the pathological findings were consistent with carcinoma; however, these findings differed from those of the stomach. We diagnosed the patient with gastric cancer of clinical stage IIA and mediastinal CUP.

To obtain a definitive tissue diagnosis and radical cure, right-sided video-assisted thoracic surgery was first performed. The mediastinal lymph node was resected, and histopathologic examination revealed a carcinoma (Figure 3(A)). We then conducted total gastrectomy, and histopathologic examination revealed an adenocarcinoma (Figure 3(B)), pathologic stage IV due to para-aortic lymph node adenopathy. These findings were distinctly different from those of the mediastinal tumor.

The patient underwent postoperative chemotherapy for residual gastric cancer. Twenty months postoperatively, he is alive with cancer.

\section{Discussion}

CUP accounts for $3 \%$ to $5 \%$ of all human cancers, is reportedly the seventh to eighth most frequent malignant tumor, and is the fourth most common cause of cancer death in both sexes [1]. CUP represents a heterogeneous group of cancer with a distinct biology and is classified depending on the patient characteristics, clinical examination findings, site involvement, and histopathological findings. CUP may be divided into subsets of favorable and unfavorable prognoses. The favorable subset includes CUP with isolated axillary nodal involvement, carcinoma with a midline distribution in young men, and others. A treatment strategy has been established in the favorable subset. Treatment response and survival are similar to those in patients with a relevant known primary tumor. In contrast, the optimal treatment for the unfavorable subset has not yet been determined. Lazaridis et al. [3] reported that the median survival of poor-prognosis CUP was 6 to 7 months.

Metastatic mediastinal lymph node CUP is rare. It is not included in the favorable subset, but its prognosis is better than that in the unfavorable subset. Hayashi [2] reported that median survival of patients with intrathoracic lymph node CUP was 28 months.

Some reports have shown the effectiveness of surgery or chemoradiotherapy for treatment of mediastinal lymph node CUP [2] [4]-[9]. Miyoshi et al. [5] reported surgical treatment for metastatic thoracic lymph node CUP. They divided their patients into two groups: those with single-station lymph node involvement (SS) and those with multiple-station lymph node involvement (MS). The SS group had a significantly better prognosis than did the MS group, and complete resection was recommended. We were able to perform complete resection of the mediastinal lymph node in our patient, but he had a poor prognosis with respect to the gastric cancer.

\section{Conclusion}

In conclusion, we have reported a rare case of single mediastinal lymph node CUP. Complete resection of a single metastatic mediastinal lymph node may be beneficial in selected patients.
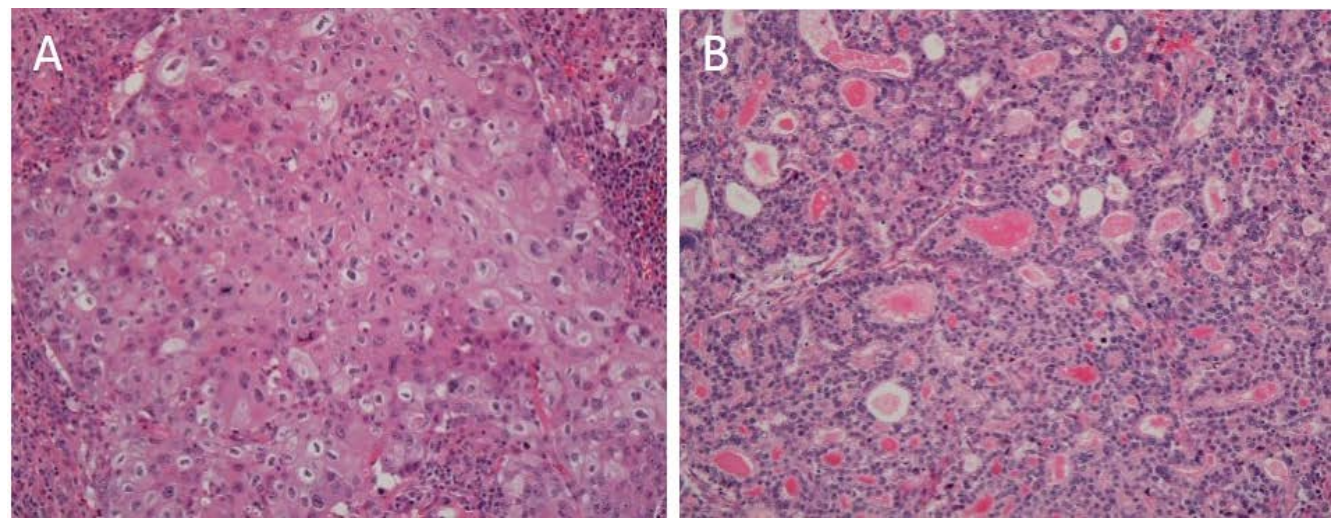

Figure 3. (A) Mediastinal lymph node: microscopic findings showed sheets of large tumor cells. The tumor cells showed no microscopic features of differentiation toward either adenocarcinoma or squamous cell carcinoma; (B) Gastric cancer: the tumor contained well-defined glandular structures with tubules. 


\section{Conflict of Interest}

The authors declare no conflict of interest.

\section{References}

[1] Pavilidis, N. and Pentheroudakis, G. (2012) Cancer of Unknown Primary Site. Lancet, 379, 1428-1435. http://dx.doi.org/10.1016/S0140-6736(11)61178-1

[2] Hayashi, K. (2007) A Case Report of a Long-Surviving Patient with Mediastinal Lymph Node Carcinoma Froma an Unknown Primary Site, with a Review of 31 Cases Reported in Japan. Annals of the Japanese Respiratory Society, 21, 624-629.

[3] Lazaridis, G., Pentheroudakis, G., Fountzilas, G. and Pavlidis, N. (2008) Liver Metastases from Cancer of Unknown Primar: A Retrospective Analysis of Presentation, Management and Prognosis in 49 Patients and Systematic Review of the Literature. Cancer Treatment Reviews, 34, 693-700. http://dx.doi.org/10.1016/j.ctrv.2008.05.005

[4] Riquet, M., Badoual, C., le Pimpec, B.F., Dujon, A. and Dane, C. (2003) Metastatic Thoracic Lymph Node Carcinoma with Unknown Primary Site. Annals of Thoracis of Surgery, 75, 244-249. http://dx.doi.org/10.1016/S0003-4975(02)04119-X

[5] Miyoshi, K., Okumura, N., Kokado, Y., Matsuoka, T., Kameyama, K., et al. (2007) Metastatic Thoracic Lymph Node Carcinoma of Unknown Origin. Japanese Journal of Lung Cancer, 47, 245-250. http://dx.doi.org/10.2482/haigan.47.245

[6] Faure, E., Riquet, M., Lombe-Weta, P.M., Hubsch, J.P. and Carnot, F. (2000) Malignant Mediastinal Lymph Node Tumors with Unknown Primary Cancers. Revue des Maladies Respiratoires, 17, 1095-1099.

[7] Miwa, K., Fujioka, S., Adachi, Y., Haruki, T., Taniguchi, Y. and Nakamura, H. (2009) Mediastinal Lymph Node Carcinoma of an Unknown Primary Site: Clinicopathological Examination. General Thoracic and Cardiovascular Surgery, 57, 239-243. http://dx.doi.org/10.1007/s11748-008-0361-5

[8] Watanabe, S., Tanaka, J., Tuboi, K, Tanaka, H., Kagamu, H., et al. (2006) A Case of Squamous Cell Carcinoma of Unknown Primary Site with Involvement of Cervicomediastinal Lymph Nodes Successfully Treated by Chemoradiotherapy. Japanese Journal of Cancer \& Chemotherapy, 33, 1943-1945.

[9] Shiota, Y., Imai, S., Sasaki, N., Tahara, K., Noma, B., et al. (2011) A Case of Mediastinal Lymph Node Carcinoma of Unknown Primary Site Treated with Docetaxel and Cisplatin with Concurrent Thoracic Radiation Therapy. Acta Medica Okayama, 65, 407-411. 
Scientific Research Publishing (SCIRP) is one of the largest Open Access journal publishers. It is currently publishing more than 200 open access, online, peer-reviewed journals covering a wide range of academic disciplines. SCIRP serves the worldwide academic communities and contributes to the progress and application of science with its publication.

Other selected journals from SCIRP are listed as below. Submit your manuscript to us via either submit@scirp.org or Online Submission Portal.
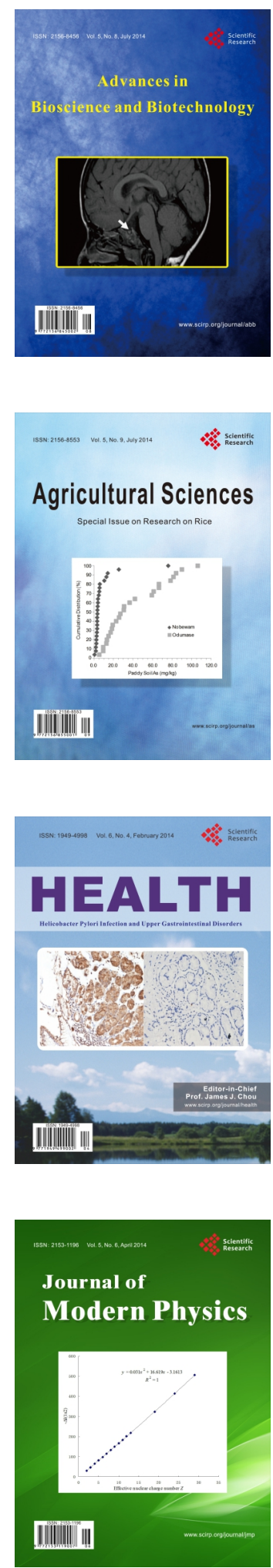
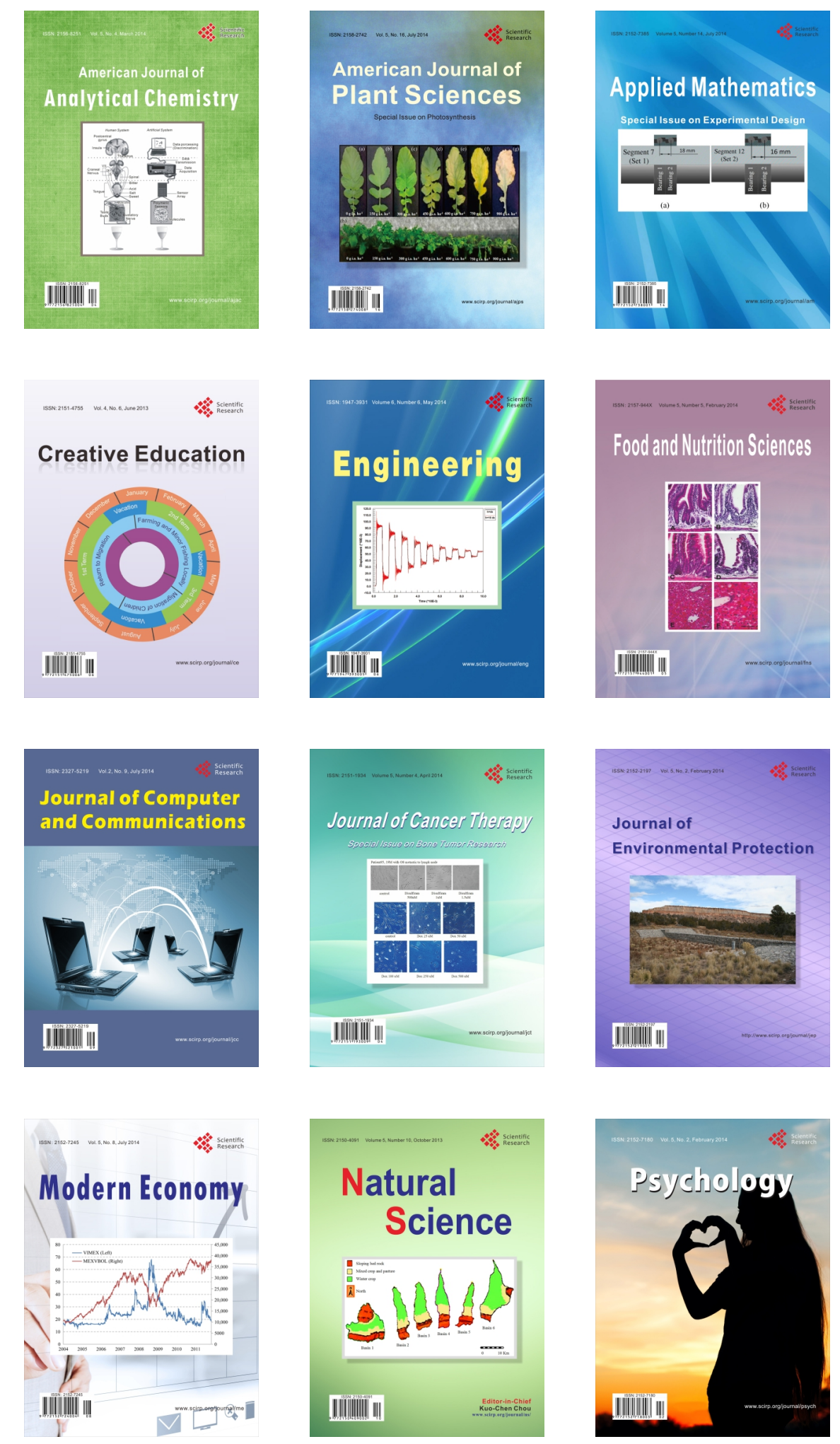